\title{
In situ mapping of chemical segregation using synchrotron x-ray imaging
}

\author{
Shikang Feng ${ }^{1}$, Enzo Liotti ${ }^{1}$, Matthew D. Wilson ${ }^{2}$, Lydia Jowitt ${ }^{2}$, Patrick S. Grant*1
}

${ }^{1}$ Department of Materials, University of Oxford, Oxford, OX1 3PH, UK

${ }^{2}$ Science and Technology Facilities Council, Rutherford Appleton Laboratory, Harwell Science and Innovation Campus, Didcot, OX11 ODE, UK

Synchrotron X-rays are a powerful tool to probe real-time changes in the microstructure of materials as they respond to an external stimulus, such as phase transformations that take place in response to a change in temperature. X-ray imaging techniques include radiography and tomography and have been steadily improved over the last decades and can now resolve micronscale or even finer structural changes in bulk specimens over timescales of a second or less. Under certain conditions, these imaging approaches can also give spatially resolved chemical information. In this brief overview of X-ray imaging, we focus on the liquid to solid transformation of metallic alloys and the temporal and spatial resolution of the accompanying segregation of alloying elements. The solidification of alloys provides an excellent case study for $\mathrm{X}$-ray imaging because it is usually accompanied by the progressive, preferential segregation of one or more of the alloying elements to either the solid or the liquid, and gives rise to surprisingly complex chemical segregation patterns. We describe chemical mapping investigations of binary and quasi-binary alloys using radiography and tomography, and recent developments in X-ray fluorescence imaging that offer the prospect of a more general, multielement mapping technique. Finally, the future developments for synchrotron-based chemical mapping are considered.

Keywords: Alloy solidification; chemical segregation; X-ray transmission; X-ray fluorescence; pixelated detector; synchrotron. 


\section{Introduction}

During the cooling and solidification of a liquid metal alloy in which the elements are initially randomly mixed, the formation of the first solid crystals from the melt generally leads to preferential segregation of one or more elements to either the new solid phase or the remaining liquid. This segregation behavior is driven by minimization of the overall free energy of the system as it transforms from liquid to a mixture of solid and liquid and finally to fully solid. The behavior described schematically by the alloy equilibrium phase diagram, which is a cornerstone of physical metallurgy. Because of this elemental redistribution, along with crystallographic anisotropy of the solid/liquid energy, convection in the liquid, and thermal gradients or perturbations, even simple two element (binary) alloys can have surprisingly complex 3D elemental distributions during solidification. In the solid state, these "frozen in" chemical segregation patterns can have a significant practical effect on alloy final properties - for example by controlling the homogeneity, type and size of second phase precipitates that form and which determine alloy ductility and toughness.

Once a few solid crystals have formed from the liquid, their morphological evolution and associated chemical segregation behavior influences the likelihood of more crystals forming as cooling continues, or that the existing crystals grow. Because the elements usually have different densities, chemical segregation and the arising concentration gradients in the liquid or solid (because diffusion is insufficiently fast to flatten these gradients) can cause buoyancy or sedimentation of the solid, and induce convective flow in the liquid. These flows can lead to macroscopic compositional inhomogeneity. For example, in $\mathrm{Al}$ alloys manufactured by direct chill casting, chemical segregation leads to a significantly higher concentration of alloying elements, and a different microstructure, near the outer surface of the casting [1]. Even at mild cooling rates $\left(0.01-5 \mathrm{Ks}^{-1}\right)$, non-equilibrium effects may arise during solidification such as composition gradients, super-saturation and the formation of metastable phases.

Before the advent of modern X-ray imaging, chemical segregation studies of high temperature phase transformations such as solidification had to be performed "post-mortem", typically by destructive sample preparation and then electron microscopy (EM) combined with different 
types of energy dispersive analysis to map chemical segregation. Detailed 3D re-constructions from sequences of 2D elemental maps obtained by sequential sectioning and EM are now relatively routine. However, EM is not well-suited to the dynamic study of some phase transformations (e.g. at high temperature) in real-time and where bulk phenomena rather than surface phenomena are important. With the advent of high flux, high energy third generation synchrotrons with highly penetrating X-rays, and the development of more efficient X-ray detectors, X-ray imaging techniques have gradually become a complementary or even preferred tool for the real-time study of many phase transformations.

\section{Element mapping using synchrotron X-ray imaging}

Extracting local element and concentration information from X-ray images is based on the principles of X-ray absorption and involves either measuring the attenuation of transmitted Xrays and/or the emitted fluorescence spectrum associated with a particular position (pixel/voxel) within a 2D (radiography) or 3D (tomography) image dataset.

In both radiography and tomography, X-rays transmitted through a sample are recorded at a 2D imaging detector, with the measured intensity (grey scale value) inversely proportional to the Xray intensity absorbed by the sample. Radiography experiments are generally carried out with a monochromatic X-ray beam and relatively thin $(<350 \mu \mathrm{m})$, constant thickness foil-like samples. In the case of a two-element binary alloy, by following a series of standard calibrations and by knowing the X-ray absorption behavior at the appropriate X-ray energy, the concentration of both elements at each pixel can be obtained [2].

A typical synchrotron radiography arrangement is shown in Figure 1 comprising a monochromatic X-ray source, a furnace to control the sample environment and a detection system. The furnace typically has one or two hot zones for isothermal or directional cooling and samples are often enclosed in a vacuum or controlled-atmosphere chamber to minimize oxidation. The acquisition system is composed of a scintillator combined with an optical system (mirror and lenses) to control the magnification and field of view, and a CCD or CMOS detector 
[3], [4]. The mirror and lens arrangement is specific to each X-ray beamline. This relatively simple arrangement provides a wide range of options for different X-ray energies, magnifications, acquisition rates, and cooling rates (up to $30 \mathrm{~K} \mathrm{~s}^{-1}$ ). High resolution 4k images at $\mathrm{kHz}$ can be achieved when used at a high flux synchrotron [5], and sub-micron resolution is possible [6].

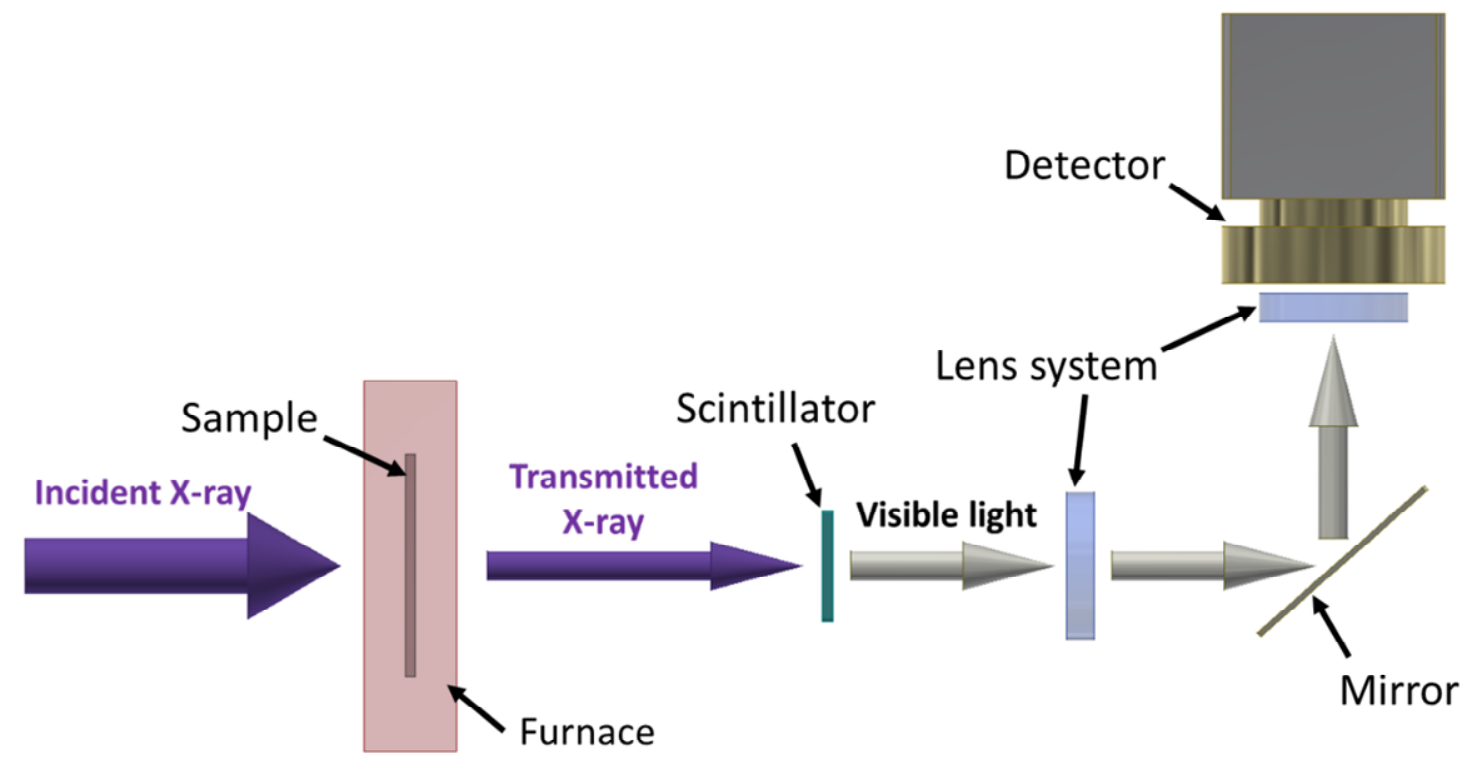

Figure 1: A schematic of a typical experimental configuration for synchrotron radiography.

However, for quantified chemical information at each pixel in the image, a key limitation is the restriction to binary or quasi-binary alloy systems. If three elements are present and form solutions with one another to some extent, unambiguous absorption contrast separation of each element is not possible. Quasi-binary systems are those alloys where the X-ray absorption coefficient of one element is significantly higher than the others (e.g. $\mathrm{Cu}$ in $\mathrm{Al}-\mathrm{Cu}-\mathrm{Si}$ [2]) at a given beam energy, so that the alloy can be treated as a binary system, although quantified distributions of each element individually are still not possible. In addition, there may also be constraints on the combination of elements. For instance, in dilute alloys where the alloying elements are next to each other in the periodic table and have similar absorption coefficients, 
such as $\mathrm{Al}$ and $\mathrm{Si}$, gaining sufficient image contrast for segregation measurement may be difficult.

X-ray tomography approaches are conceptually equivalent to radiography but the sample is typically a cylinder that is rotated while 2D projections are recorded. More details on the reconstruction of tomographs from 2D radiographic projections and the evaluation of the absorption line integral can be found elsewhere [7]. For chemical measurements, care is needed that the elemental distributions do not change significantly over the period of a single rotation. This is normally not an issue for solid phases, but if, for example, short timescale convection effects in the liquid are of interest, distributions may change significantly within a scan rotation, even at tomography acquisition speeds of up to $10 \mathrm{~Hz}$. At high acquisition rates, centripetal forces might also affect or promote radial chemical segregation in liquid phases.

$\mathrm{X}$-ray fluorescence microscopy (XFM) is an alternative to absorption contrast-based techniques in which a sample surface is raster-scanned through a focused primary X-ray beam and the induced fluorescence X-rays (XRF) at each $\mathrm{x}-\mathrm{y}$ position are measured using an XRF spectrometer [8], [9]. A composite image is formed by summing the counts at discrete XRF energies for each element in the sample, for each position in the scan. At synchrotrons, XFM can be performed with nano- $(\sim 10-500 \mathrm{~nm})$ or micro- $(1-10 \mu \mathrm{m})$ focused beams and collection times can be lower than $1 \mathrm{~ms}$ per point [10]. Figure 2(a) shows a typical XFM configuration with a monochromatic X-ray source. A radiography transmission camera can also be used to aid alignment and provide complimentary imaging information. To increase count rates, a relatively large area, pixelated XRF detector array (e.g. comprising up to a $20 \times 20$ array of $1 \mathrm{~mm}^{2}$ individual detectors) is placed close to the sample to capture as much of the isotropic XRF emission as is practical [11].

An alternative approach is full field of view (FoV) XRF that uses an unfocused X-ray white beam source and collimates the fluorescence signal, for example by a pinhole as in Figure 2(b), to project the signal onto a pixelated energy dispersive imaging detector (EDI) that captures a 2D "image" in a single exposure. In principle, the significant advantage of XRF imaging over radiography is that because each pixel of the detector records a spectrum of photon energies (i.e. 


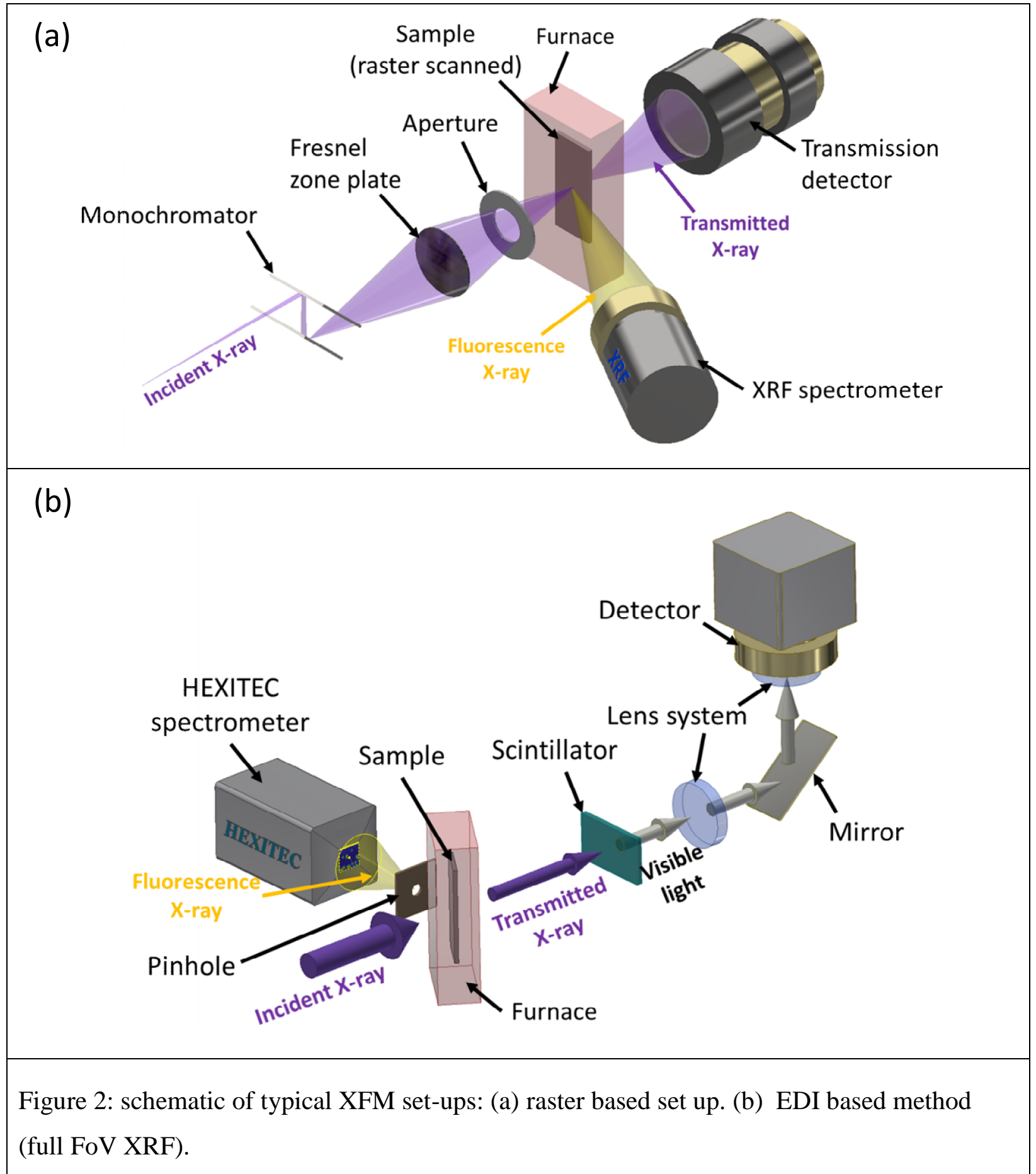

the detector is a pixelated spectrometer), the concentration of many elements can be deduced at each pixel simultaneously, allowing chemical segregation evolution in complex composition alloys to be studied. Although various types of EDI detector are available, the technique is at an earlier stage of development compared with radiography, tomography and XFM. The main challenges include ensuring a sufficiently large, useful photon flux arriving at each pixel, self- 
absorption effects within the sample, and a spatial resolution currently limited to $\sim 10 \mu \mathrm{m}$ or more.

\section{X-ray radiography mapping of chemical segregation}

In this section, we provide examples representative of chemical segregation studies during solidification using radiography. The focus is on $\mathrm{Al}$ alloys, which have been the most studied because (i) their melting points are relatively low $\left(\sim 660^{\circ} \mathrm{C}\right)$, (ii) a number of binary systems offer good X-ray adsorption contrast, and (iii) they form the basis of useful commercial alloys e.g. Al-Cu forms the basis of the commercial 2000 alloy series alloy. The studies can be categorized in three groups: morphological stability, growth of columnar dendritic grains, and the formation and growth of equiaxed grains and intermetallic phases.

The transformation of a planar solid/liquid interface into more morphologically complex cells or dendrites is initiated by thermal and/or concentration inhomogeneity along the solid-liquid front during growth [12]-[16]. Buffet et al. studied $\mathrm{Cu}$ segregation generated by a planar solidification front in a uni-directionally solidifying Al-4wt\%Cu alloy [12]. Figures 3(a) and (b) show radiographs of the solid/liquid planar front growing upwards under an imposed cooling rate of $0.008 \mathrm{Ks}^{-1}$. Because the $\alpha-\mathrm{Al}$ solid phase has a $\mathrm{Cu}$ concentration lower than the liquid from which it is forming (as determined by the equilibrium phase diagram), the "excess" $\mathrm{Cu}$ is rejected into the liquid ahead of the front and forms a $\mathrm{Cu}$-rich diffusion field. Figure 3(d) shows the $\mathrm{Cu}$ concentration in the liquid was at a maximum at the solid/liquid interface, and decreased with distance from the interface to the nominal alloy composition. Figure 3(c) shows that at some point, the build-up of $\mathrm{Cu}$ at the interface caused the planar interface to become unstable and to transition to a more complex dendritic morphology. It is worth noting that at true thermodynamic equilibrium, neither solid nor liquid phase should show any chemical segregation - whereas even at this modest cooling rate of $0.008 \mathrm{Ks}^{-1}$, chemical segregation was distinct - because diffusion was insufficiently fast to flatten the chemical gradients as they evolved. 

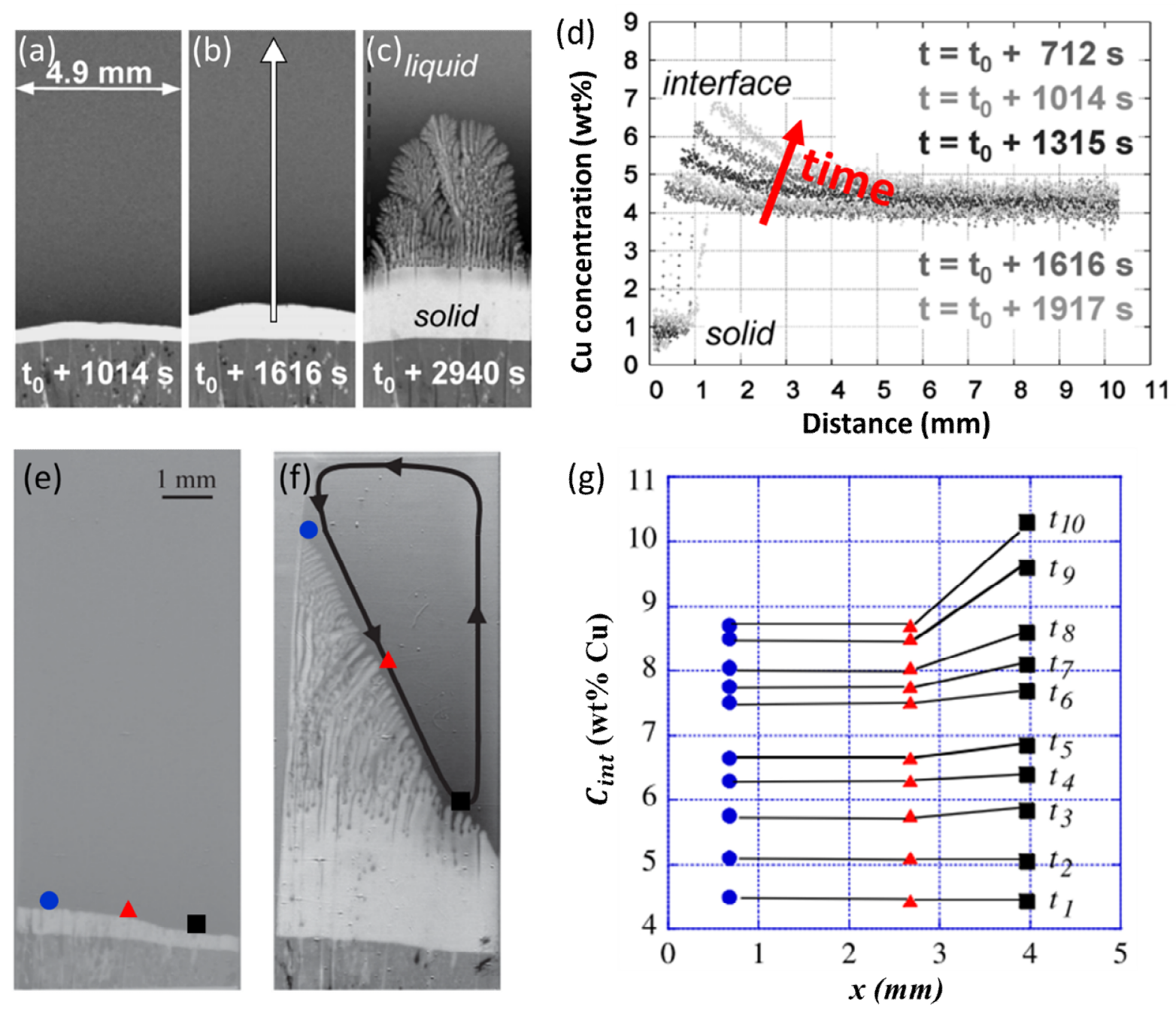

Figure 3: (a) and (b) Radiographs showing solid $\alpha$-Al growing with a planar interface during the early stages of the uni-directional solidification of $\mathrm{Al}-4 \mathrm{Cu}$ at $0.008 \mathrm{Ks}^{-1}$; $\mathrm{t}_{0}$ is the instant when solidification started [12]. (c) A radiograph showing the development of a dendritic microstructure due to increasing interfacial instability [12]. (d) Solute profiles along the white arrow in (b) measured at different times during solidification [12]. (e) and (f) Radiographs showing preferential growth induced by solute segregation during directional solidification of $\mathrm{Al}-4 \mathrm{Cu}$ at $0.005 \mathrm{Ks}^{-1}$, at $\mathrm{t} \approx 17 \mathrm{~min}$ and $\mathrm{t} \approx 88 \mathrm{~min}$ respectively. The arrows in (f) indicate liquid flow [14]. (g) Evolution of liquid compositions at the solid/liquid interface at $x=0.7$ $\mathrm{mm}, x=2.6 \mathrm{~mm}$ and $x=4 \mathrm{~mm}$ (as indicated in (e) and (f)) for times $\mathrm{t}_{1}=20$ min and $\mathrm{t}_{2}=40$ $\min$ and then to $t_{10}=360 \mathrm{~min}$ at $40 \mathrm{~min}$ intervals [14].

Using the same alloy, Bogno et al. [14] investigated how concentration differences in the liquid from chemical segregation at the solid/liquid interface can lead to convection; Figures 3(e) and (f) show the resulting microstructural instability at a cooling rate of $0.005 \mathrm{Ks}^{-1}$. The slight 
preferential growth of solid on the left side of the radiograph in Figure 3(e) slightly increased the lateral ( $x$-direction) solute gradient, as shown in Figure 3(g). As growth continued and the solid/liquid interface transitioned to a more cellular/dendritic form, continuing $\mathrm{Cu}$ rejection led to macro-scale recirculation flows (Figure 3(f)) and a range of growth rates across the dendrite front. Radiographic videos have also shown significant solute flow in the liquid ahead of a growing dendrite array [17], because of spatially varying liquid density differences due to chemical segregation. The imaged concentration fluctuations due to convection were correlated to oscillations in the dendrite tip growth rate - an effect that is likely more pronounced in large castings where significant long-range convection can occur.

Finer-scale chemical segregation during columnar dendritic growth and its effect on microstructural development has also been resolved by X-ray imaging [2], [17]-[19]. Mathiesen et al. measured the solute concentration around dendrite tips and within the liquid channels between dendrites in directionally solidified $\mathrm{Al}-30 \mathrm{wt} \% \mathrm{Cu}$ at $0.6 \mathrm{Ks}^{-1}$ [17]. Figure 4(a) is a radiograph of three $\mathrm{Al}$ dendritic arms surrounded by $\mathrm{Cu}$-enriched liquid, shown more clearly in the corresponding false-color contour map of $\mathrm{Cu}$ concentration in Figure 4(b). There was increased chemical segregation "deeper" into the mushy zone (decreasing liquid fraction).

By combining this type of micron-scale and $>1 \mathrm{~Hz}$ resolution, chemical segregation effects on the fragmentation of growing dendrites has been studied because of its potential exploitation to provide a beneficial grain refining effect in commercial alloys [3], [18], [20]-[22]. For example, Ruvalcaba et al. studied a solidifying $\mathrm{Al}-20 \mathrm{wt} \% \mathrm{Cu}$ alloy at $1.2 \mathrm{Ks}^{-1}$ and the radiograph sequence in Figure 4(c) shows the detachment of a tertiary dendrite arm (in the white circle) [18]. Corresponding time-resolved contour maps of $\mathrm{Cu}$ concentration in the fragmentation region (Figure 4(d)) revealed fine-scale $\mathrm{Cu}$ enrichment around the fracturing root of the tertiary arm. Liotti et al. [20] then used detailed solute gradient and fragmentation measurements deep in the mushy zone of an Al-25wt\% Cu alloy solidified at $1 \mathrm{Ks}^{-1}$ (Figure 4(e)) to show that the local solute concentration gradient controlled the fragmentation rate (Figure 4(f)): steep chemical gradients in the liquid created an environment in which any morphological perturbation to the solid-liquid interface, for example due to convection, was more likely to lead to destabilization and local re-melting of regions of relatively high curvature i.e. at the dendrite roots. 

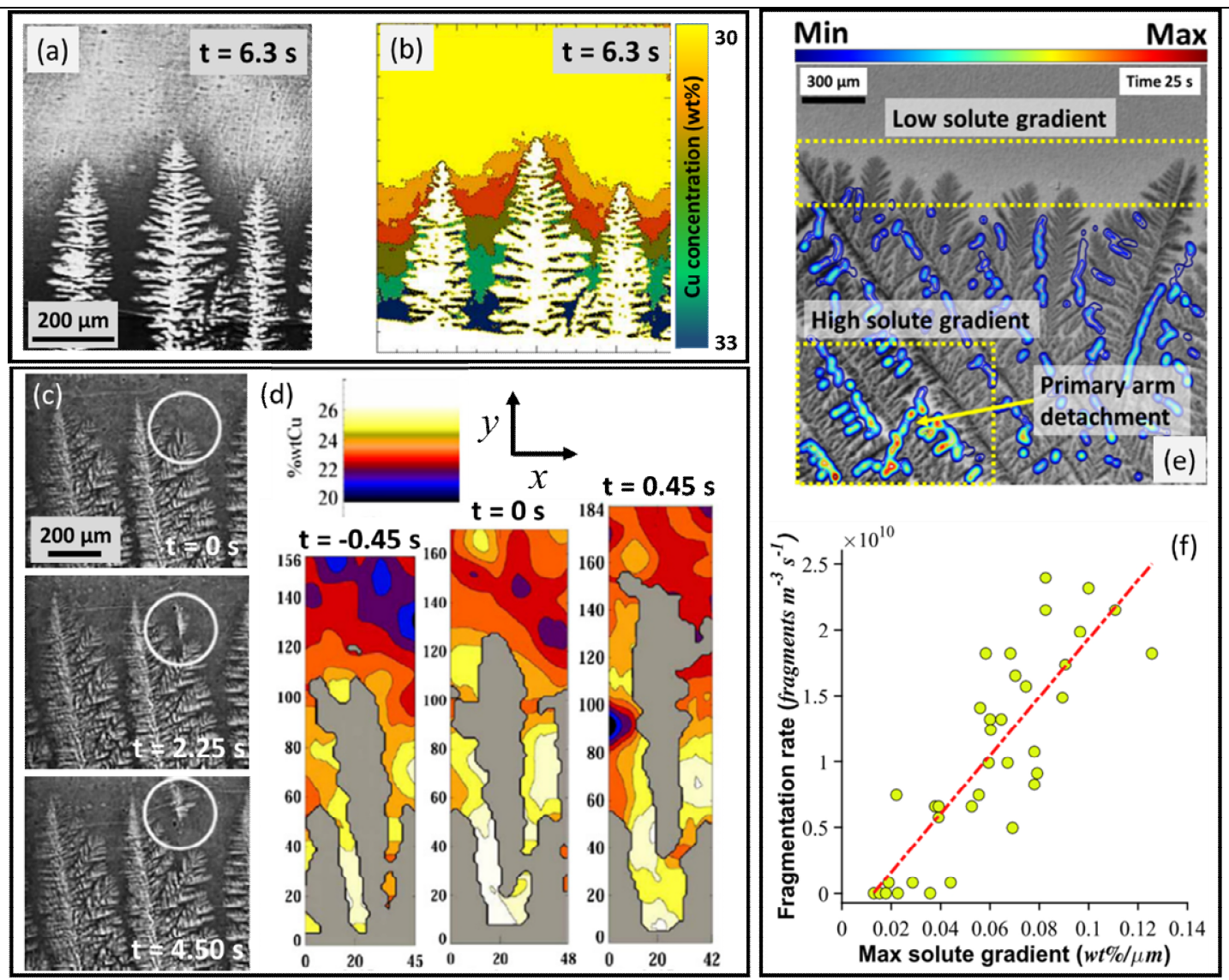

Figure 4: (a) A radiograph of three $\alpha$-Al columnar dendrites solidifying in an Al-30Cu alloy at $0.6 \mathrm{Ks}^{-1}$ [17]. (b) The corresponding $\mathrm{Cu}$ concentration contour map [17]. (c) A radiograph sequence showing dendrite fragmentation (in the white circle) during the solidification of an Al-20Cu alloy at $1.2 \mathrm{Ks}^{-1}$ [18]. (d) The corresponding $\mathrm{Cu}$ concentration contour map with the $x-y$ scale given in $\mu \mathrm{m}$. Time $\mathrm{t}=0 \mathrm{~s}$ was set when detachment of the fragment was first observed [18]. (e) Horizontal solute gradient contour map within the inter-dendritic channels of a solidifying Al-25Cu alloy at $1 \mathrm{Ks}^{-1}$ [20]. (f) The local dendrite fragmentation rate as a function of the maximum local inter-dendritic solute gradient in the liquid. The red dashed line shows a linear best-fit [20].

The use of radiography-based chemical segregation mapping has also been applied to equiaxed solidification [23]-[26], which occurs when the liquid is undercooled below the alloy equilibrium freezing temperature before solidification starts, and the nucleation of solid that forms a new crystal is facilitated by extrinsic, solid micron-scale motes or "grain refiners", deliberately added (inoculated) to the melt. Such conditions predominate in the casting of most commercial Al alloys. Figure 5(a) shows radiographs of a solidifying Al-10wt\%Cu alloy at 0.008 $\mathrm{Ks}^{-1}$, inoculated with $\mathrm{TiB}_{2}$ particles, and Figures 5(b) and (c) provide zoomed-in views around the newly-formed equiaxed, dendritic crystal labelled "D1" [25]. The chemical profile ahead of 
the tip of D1 at two instants is shown in Figure 5(d). The liquid $\mathrm{Cu}$ concentration between D1 and a neighboring crystal increased steadily with time (and as the temperature decreased and the crystal grew), and eventually the two solute fields started to overlap, which caused a sharp decrease in the growth velocity of the crystals and restricted their final size.

Liotti et al. studied the effect of cooling rate and solute concentration on the nucleation of equiaxed $\alpha-\mathrm{Al}$ crystals in a range of $\mathrm{Al}-\mathrm{Cu}$ alloys inoculated with $\mathrm{TiB}_{2}$ particles [23]. Combining a "reverse time" approach with machine learning, they used instantaneous measurements of the $\mathrm{Cu}$ concentration from a cluster of pixels just before a new $\alpha$-Al crystal appeared at that position to estimate automatically the formation undercooling of more than 14,000 separate crystal formation events. With this comparatively large data-set they were able to analyze crystal formation events not only spatially but also in the time domain, showing that new crystals did not form more or less continuously as the temperature reduced and as commonly assumed, but formed in distinct "waves" or bursts. Figures 5(e)-(g) show three formation waves (denoted as 1, 2 and 3) in a solidifying $\mathrm{TiB}_{2}$-inoculated $\mathrm{Al}-25 \mathrm{wt} \% \mathrm{Cu}$ alloy at $0.3 \mathrm{Ks}^{-1}$. For each wave, solute rejected from a band of crystals created a solute-rich region in which further solid nucleation and crystal formation was temporarily suppressed. As cooling proceeded, the additional undercooling to overcome the solute-suppression was eventually achieved, at which point, since nucleation rate is non-linearly related to undercooling [27], a burst of new crystals appeared almost simultaneously across the field of view, with a time constant of repetition related to the solidification conditions [23].

Switching from primary $\alpha$-Al crystal formation to secondary phases, Feng et al. investigated the formation of $\mathrm{Al}_{13} \mathrm{Fe}_{4}$, one of the most commonly formed Fe-rich intermetallic compounds (IMCs) in commercial $\mathrm{Al}$ alloys [28]. Figure 5(h) shows a radiograph of primary $\mathrm{Al}_{13} \mathrm{Fe}_{4}$ crystals forming as the first phase in an Al-3wt\% Fe alloy at $0.5 \mathrm{Ks}^{-1}$. Figure 5(i) is a plot of the Fe concentration in front of the IMC labelled "A", along the red arrow labelled in Figure 5(h). In this case, rather than solute enrichment in the liquid, there was solute (Fe) depletion in the local liquid to feed the growing Fe-rich IMC crystals (Figure 5(i)). These measurements were used to understand the relationship between solidification parameters and the formation rate of the 

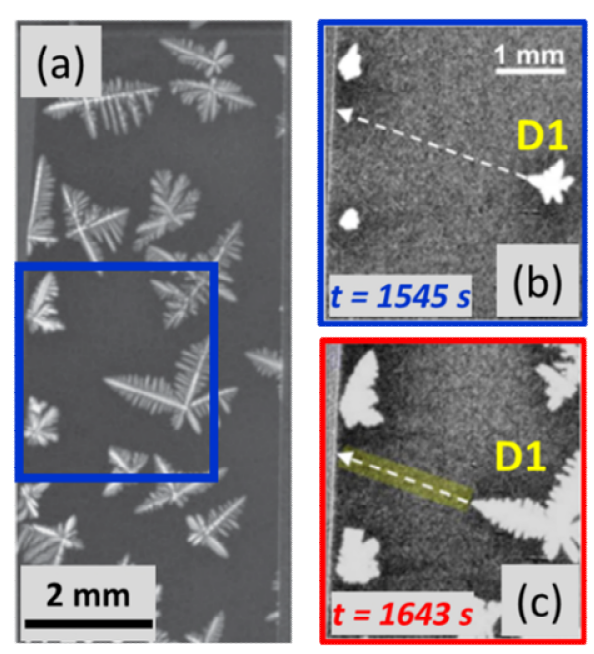

(d)
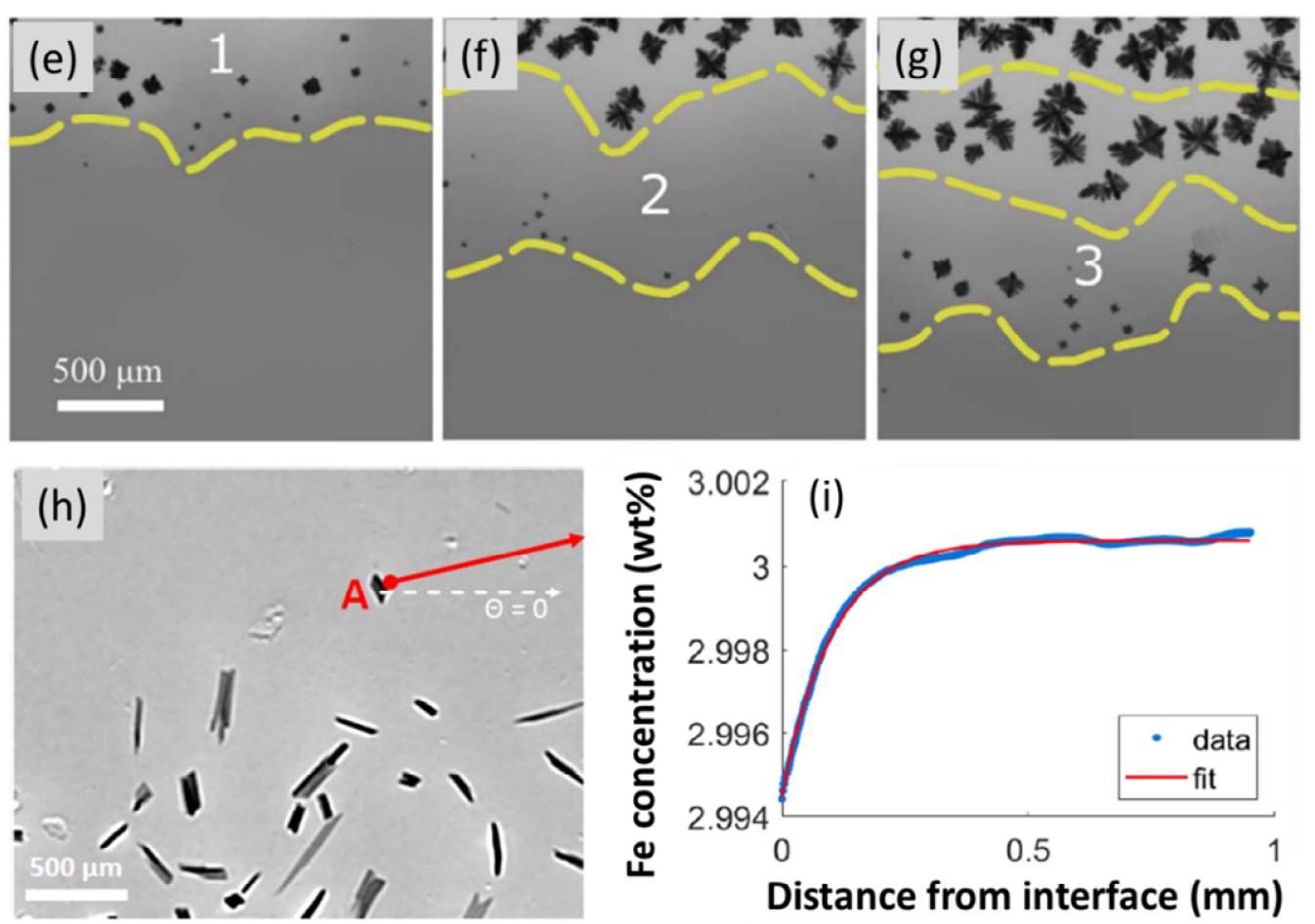

Figure 5: (a) A radiograph of equiaxed $\alpha$-Al dendrites growing in an Al-10Cu alloy inoculated with $\mathrm{TiB}_{2}$ particles at $0.008 \mathrm{Ks}^{-1}$ [25]. (b) and (c) Zoomed-in images around crystal D1 at time $\mathrm{t}=1545 \mathrm{~s}$ and $\mathrm{t}=1643 \mathrm{~s}$ [25]. (d) $\mathrm{Cu}$ concentration measured as a function of distance ahead of the dendrite tip of D1 along the direction indicated by the white dashed arrow in (b) and (c). $C_{0}$ denotes the bulk alloy composition [25]. (e)-(g) A radiograph sequence showing formation of equiaxed $\alpha$-Al crystals in an Al-25Cu alloy inoculated with $\mathrm{TiB}_{2}$ particles at $0.3 \mathrm{Ks}^{-1}$, at times $\mathrm{t}=16 \mathrm{~s}, 29 \mathrm{~s}$ and $38 \mathrm{~s}$ respectively $(\mathrm{t}=0$ was set to the frame when the first crystal appeared in the field of view) [23]. (h) A radiograph of primary $\mathrm{Al}_{13} \mathrm{Fe}_{4}$ crystals in a solidifying Al-3Fe alloy at $0.5 \mathrm{Ks}^{-1}$ [28]. (i) The solute profile with a best-fit to theory in [29], along the direction indicated by the red arrow in (h) [28]. 
IMCs, which in turn controlled the final number and therefore size of the IMCs, a key parameter in controlling alloy properties such as ductility.

\section{Solute mapping using synchrotron tomography}

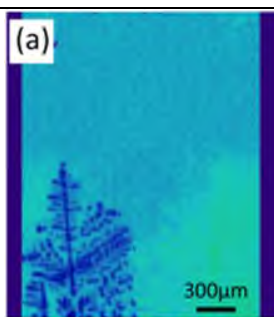

(a) $t=76 \mathrm{~s}$

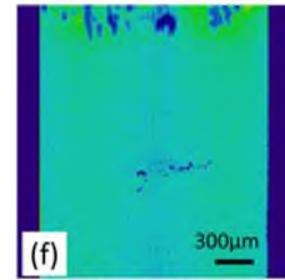

(f) $t=76 \mathrm{~s}$

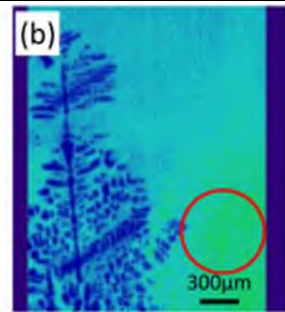

(b) $t=166 \mathrm{~s}$

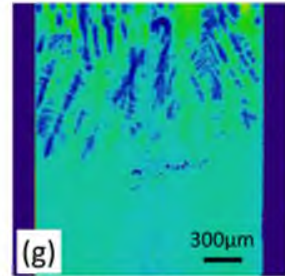

(g) $\mathrm{t}=166 \mathrm{~s}$

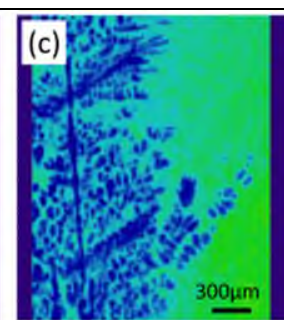

(c) $\mathrm{t}=346 \mathrm{~s}$

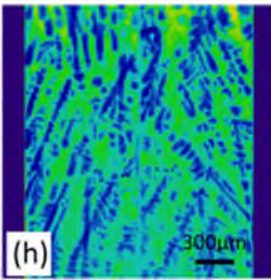

(h) $t=346 \mathrm{~s}$

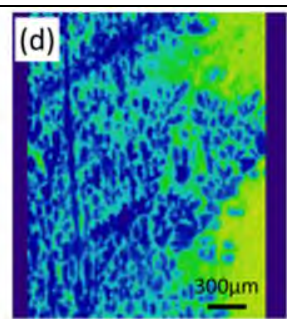

(d) $\mathrm{t}=796 \mathrm{~s}$

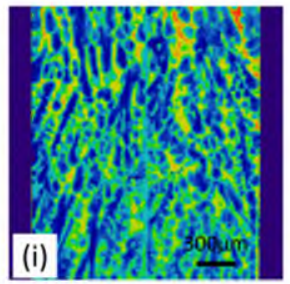

(i) $\mathrm{t}=796 \mathrm{~s}$

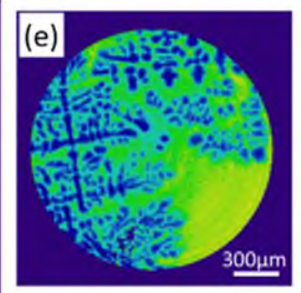

(e) $\mathrm{t}=346 \mathrm{~s}$, Transverse

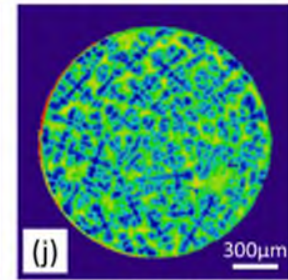

(j) $\mathrm{t}=346 \mathrm{~s}$, Transverse $<5.5 \mathrm{Cu}(\mathrm{wt} \%) \quad 33>$

Figure 6: Time-resolved vertical 2D slices from 3D tomographs showing the $\mathrm{Cu}$ concentration during (a)-(d) upward solidification and (f)-(i) downward solidification of an Al-15Cu alloy at $0.05 \mathrm{Ks}^{-1}$. (e) Transverse slice at $\mathrm{t}=346 \mathrm{~s}$ for upward solidification. (j) Transverse slice at $\mathrm{t}=$ $346 \mathrm{~s}$ for downward solidification [31].

Radiography is limited to thin, foil-like specimens that may limit the applicability of insights to real problems. In contrast, X-ray tomography in principle allows the dynamics of solidification and other phase transformations to be resolved in 3D. However the analysis of the data is more complicated and cumbersome and while qualitative imaging by tomography can be highly instructive, there are relatively few reports of quantified chemical segregation studies by X-ray tomography [30], [31]. Nelson et al. developed a method to extract 3D solute distributions from $\mathrm{X}$-ray tomographs of a solidifying $\mathrm{Al}-15 \mathrm{wt} \% \mathrm{Cu}$ alloy during directional solidification at $0.05 \mathrm{Ks}^{-}$ ${ }^{1}$, both parallel and anti-parallel to gravity [31]. A sequence of $2 \mathrm{D}$ slices of the $3 \mathrm{D} \mathrm{Cu}$ distribution is shown in Figure 6. When solidified upwards (Figures 6(a)-(d)), Cu segregated radially towards the right side of the field of view; when solidified downwards (Figures 6(f)-(i)) 
there was practically no radial $\mathrm{Cu}$ macro-segregation because rejected $\mathrm{Cu}$ drained downwards, out of inter-dendritic regions and was able to be mixed with the remaining liquid (compare Figures 6(e) and (j)).

\section{Multi-element mapping combining radiography and fluorescence spectroscopy}

X-ray fluorescence spectroscopy techniques have only recently been applied to investigate chemical segregation phenomena during solidification, exploiting the potential - unlike radiography and tomography - to map multiple elements simultaneously. For example, Kobayashi et al. used a simultaneous radiography-fluorescence to obtain the partition coefficient of each element at a planar solid/liquid interface during the high temperature solidification of a complex Fe-Cr-Ni-Mo-Cu alloy - data that was otherwise unobtainable by theory or experiment $[32]$.

Liotti et al. developed a synchrotron-based radiography-fluorescence technique for elemental imaging in which EDI detectors were used to map simultaneously multi-element segregation during the solidification of an Al-Cu-Ag-Zr-Mo alloy [33]. In order to assess the potential of this approach further, the authors have recently applied it to a model Al-23Pt-20Er (wt\%) alloy designed to produce chemical segregation behavior that would best match the characteristics of the HEXITEC EDI detector [34]. Figure 7(a) is a sequence of radiographs and separate X-ray fluorescence Pt and Er maps showing the formation of $\mathrm{Al}_{24} \mathrm{Pt}_{9} \mathrm{Er}_{4}(\mathrm{APE})$ and $\mathrm{Al}_{3} \mathrm{Er}(\mathrm{AE})$ intermetallic phases from the liquid during solidification of the Al-23Pt-20Er alloy at $0.1 \mathrm{Ks}^{-1}$. Figures 7(b) and (c) provide examples of how this type of XRF image data can be used, plotting the row-wise ( $x$-direction in the XRF images) summation of pixel fluorescence intensity as a function of $y$-position and temperature. On the initial formation of the Pt-rich APE phase, the surrounding liquid was depleted in Pt while the Er distribution was largely unchanged; when the Er-rich $\mathrm{AE}$ phase formed later at lower temperature, the $\mathrm{Er}$ in the surrounding liquid was then similarly depleted. This example confirms the potential to map chemical segregation of multiple elements in situ simultaneously using EDI detectors. 


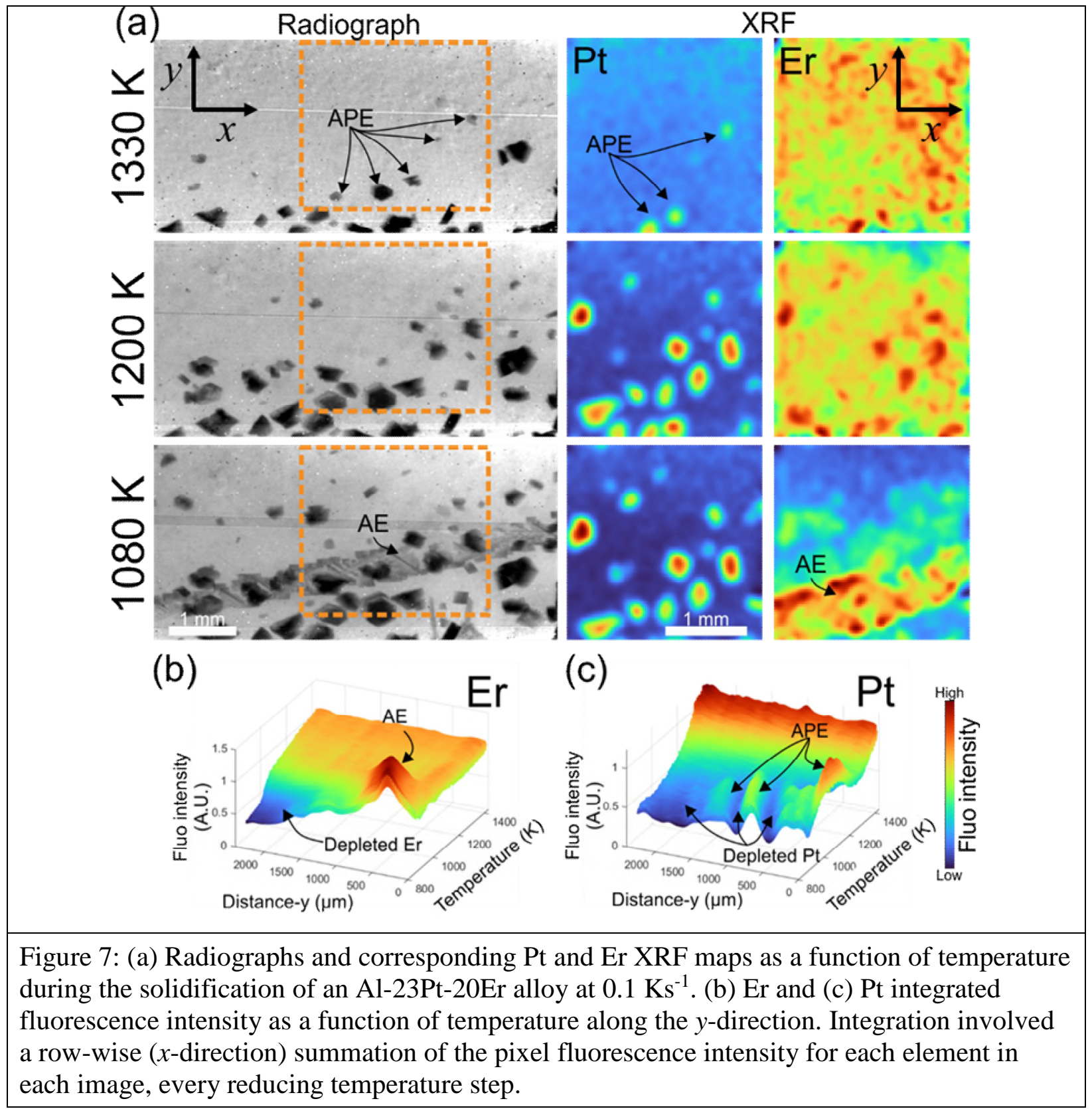

\section{Challenges and Opportunities}

Synchrotron-based X-ray radiography and tomography are now capable of mapping elements at the micron-scale and with sufficient temporal resolution to resolve the dynamics of chemical segregation during metallic alloy solidification - in some cases at cooling rates typical of 
industrial processes. The insights gained are helping to understand commercial processes and can also be used to estimate parameters and variables otherwise difficult to obtain, such as partition coefficients and diffusion coefficients. However, increased temporal resolution - towards $\mathrm{kHz}$ frequencies - is needed to map chemical segregation in important processes that operate further from equilibrium and where radiographic images are revealing new insights, for example in laser welding [35], [36] and additive manufacturing [37]-[39]. Synchrotrons around the world undergo periodic upgrade programmes and current upgrade activities offer the prospect of increasing X-ray fluxes at the sample by $10-1,000$ times. These upgrades offer the prospect of resolving the dynamics of chemical segregation in further from equilibrium processes [40], [41]. However, the increased X-ray flux will also require faster detectors and data handling, and different furnace rig and sample designs e.g. to avoid uncontrolled sample heating effects. In addition, stronger departures from equilibrium induced, for example by faster heating and/or cooling effects, imply diffusion distances over which chemical segregation can occur will be restricted and therefore the spatial resolution provided by the optical set-up will also need to be increased.

For multi-element mapping by XFM, increases in X-ray flux will enable better sensitivity and reduced scan times, which may then become simply limited by sample raster speeds and detector count rates [42]. For full FoV XRF imaging with EDI detectors, detection integration times may be reduced to $1-10 \mathrm{~ms}$, and by tiling the EDI detectors, it will be possible to image larger areas without losing temporal resolution [43]. Although full FoV XRF imaging is unlikely to achieve the spatial resolution of conventional XFM, the ability to investigate large areas relatively quickly - without moving the sample - ensures the further development of EDI detectors and new applications.

Across all X-ray imaging techniques, ever increasing spatio-temporal resolution will continue to increase data volumes that must be stored and analyzed: even relatively short synchrotron-based experimental campaigns already generate more data than can be analyzed by a large team of researchers. Where systematic, quantified data is to be extracted from terra-bytes of qualitative images, automated analysis and extraction techniques based on machine learning and artificial intelligent are increasingly important [23]. 


\section{Acknowledgement}

The authors would like to thank Thomas Connolley (Diamond Light Source) for his help with radiography-fluorescence experiments and comments on the manuscript. The authors would also like to thank UK Engineering and Physical Sciences Research Council for funding under grant number EP/N007638/1 (Future Liquid Metal Engineering Hub).

\section{References}

[1] A. V. Reddy and N. C. Beckermann, "Modeling of macrosegregation due to thermosolutal convection and contraction-driven flow in direct chill continuous casting of an Al-Cu round ingot," Metall. Mater. Trans. B, vol. 28, no. 3, pp. 479-489, Jun. 1997.

[2] W. U. Mirihanage, K. V. Falch, I. Snigireva, A. Snigirev, Y. J. Li, L. Arnberg, and R. H. Mathiesen, "Retrieval of three-dimensional spatial information from fast in situ twodimensional synchrotron radiography of solidification microstructure evolution," Acta Mater., vol. 81, pp. 241-247, Dec. 2014.

[3] E. Liotti, A. Lui, R. Vincent, S. Kumar, Z. Guo, T. Connolley, I. P. Dolbnya, M. Hart, L. Arnberg, R. H. Mathiesen, and P. S. Grant, "A synchrotron X-ray radiography study of dendrite fragmentation induced by a pulsed electromagnetic field in an $\mathrm{Al}-15 \mathrm{Cu}$ alloy," Acta Mater., vol. 70, pp. 228-239, May 2014.

[4] R. H. Mathiesen, L. Arnberg, H. Nguyen-Thi, and B. Billia, "In Situ X-Ray Video Microscopy as a Tool in Solidification Science," JOM, vol. 64, no. 1, pp. 76-82, Jan. 2012.

[5] R. Mokso, C. M. Schlepütz, G. Theidel, H. Billich, E. Schmid, T. Celcer, G. Mikuljan, L. Sala, F. Marone, N. Schlumpf, and M. Stampanoni, "GigaFRoST: The gigabit fast readout system for tomography," J. Synchrotron Radiat., vol. 24, no. 6, pp. 1250-1259, 2017.

[6] T. Kameshima, A. Takeuchi, K. Uesugi, T. Kudo, Y. Kohmura, K. Tamasaku, K. Muramatsu, T. Yanagitani, M. Yabashi, and T. Hatsui, "Development of an X-ray imaging detector to resolve $200 \mathrm{~nm}$ line-and-space patterns by using transparent ceramics layers bonded by solid-state diffusion," Opt. Lett., vol. 44, no. 6, p. 1403, Mar. 2019.

[7] J. (Jens) Als-Nielsen and D. McMorrow, Elements of modern X-ray physics. Wiley, 2011.

[8] S. Barkan, V. D. Saveliev, Y. Wang, L. Feng, E. V Damron, and Y. Tomimatsu, "Extreme High Count Rate Performance with a Silicon Drift Detector and ASIC Electronics," in Biological and Chemical Research, 2015, vol. 2015, no. July, pp. 338-344.

[9] N. Tartoni, R. Crook, T. Krings, D. Protić, C. Ross, L. Bombelli, R. Alberti, T. Frizzi, and V. Astromskas, "Monolithic multi-element HPGe detector equipped with CMOS preamplifiers: Construction and characterization of a demonstrator," IEEE Trans. Nucl. Sci., vol. 62, no. 1, pp. 387-394, 2015.

[10] C. Vanhoof, J. R. Bacon, A. T. Ellis, U. E. A. Fittschen, and L. Vincze, "2019 atomic spectrometry update-A review of advances in X-ray fluorescence spectrometry and its special applications," J. Anal. At. Spectrom., vol. 34, no. 9, pp. 1750-1767, Sep. 2019.

[11] C. G. Ryan et al., "Maia X-ray fluorescence imaging: Capturing detail in complex natural samples," J. Phys. Conf. Ser., vol. 499, no. 1, 2014.

[12] A. Buffet, H. N. Thi, A. Bogno, T. Schenk, N. Mangelinck-Noel, G. Reinhart, N. 
Bergeon, B. Billia, and J. Baruchel, "Measurement of solute profiles by means of synchrotron X-ray radiography during directional solidification of Al-4 wt\% Cu alloys," Mater. Sci. Forum, vol. 649, no. January, pp. 331-336, 2010.

[13] B. Billia, H. Nguyen-Thi, N. Mangelinck-Noel, N. Bergeon, H. Jung, G. Reinhart, A. Bogno, A. Buffet, J. Hartwig, J. Baruchel, and T. Schenk, "In Situ Synchrotron X-ray Characterization of Microstructure Formation in Solidification Processing of Al-based Metallic Alloys," ISIJ Int., vol. 50, no. 12, pp. 1929-1935, Dec. 2010.

[14] A. Bogno, H. Nguyen-Thi, A. Buffet, G. Reinhart, B. Billia, N. Mangelinck-Noël, N. Bergeon, J. Baruchel, and T. Schenk, "Analysis by synchrotron X-ray radiography of convection effects on the dynamic evolution of the solid-liquid interface and on solute distribution during the initial transient of solidification," Acta Mater., vol. 59, no. 11, pp. 4356-4365, 2011.

[15] T. Schenk, H. Nguyen Thi, J. Gastaldi, G. Reinhart, V. Cristiglio, N. Mangelinck-Noël, H. Klein, J. Härtwig, B. Grushko, B. Billia, and J. Baruchel, “Application of synchrotron Xray imaging to the study of directional solidification of aluminium-based alloys," J. Cryst. Growth, vol. 275, no. 1, pp. 201-208, 2005.

[16] Y. Chen, H. Nguyen-Thi, D. Z. Li, A.-A. Bogno, B. Billia, and N. M. Xiao, "Influence of natural convection on microstructure evolution during the initial solidification transient: comparison of phase-field modeling with in situ synchrotron X-ray monitoring data," IOP Conf. Ser. Mater. Sci. Eng., vol. 33, no. 1, p. 012102, Jul. 2012.

[17] R. H. Mathiesen and L. Arnberg, "X-ray radiography observations of columnar dendritic growth and constitutional undercooling in an Al-30wt\%Cu alloy," Acta Mater., vol. 53, no. 4, pp. 947-956, Feb. 2005.

[18] D. Ruvalcaba, R. H. Mathiesen, D. G. Eskin, L. Arnberg, and L. Katgerman, "In situ observations of dendritic fragmentation due to local solute-enrichment during directional solidification of an aluminum alloy," Acta Mater., vol. 55, no. 13, pp. 4287-4292, Aug. 2007.

[19] W. U. Mirihanage, K. V. Falch, D. Casari, S. McFadden, D. J. Browne, I. Snigireva, A. Snigirev, Y. J. Li, and R. H. Mathiesen, "Non-steady 3D dendrite tip growth under diffusive and weakly convective conditions," Materialia, vol. 5, p. 100215, Mar. 2019.

[20] E. Liotti, A. Lui, S. Kumar, Z. Guo, C. Bi, T. Connolley, and P. S. Grant, "The spatial and temporal distribution of dendrite fragmentation in solidifying Al-Cu alloys under different conditions," Acta Mater., vol. 121, pp. 384-395, 2016.

[21] R. H. Mathiesen, "Stray crystal formation in Al-20wt.\% Cu studied by synchrotron X-ray video microscopy," Mater. Sci. Eng. A, vol. 413, pp. 283-287, 2005.

[22] R. H. Mathiesen, L. Arnberg, P. Bleuet, and A. Somogyi, "Crystal fragmentation and columnar-to-equiaxed transitions in Al-Cu studied by synchrotron X-ray video microscopy," Metall. Mater. Trans. A, vol. 37, no. 8, pp. 2515-2524, Aug. 2006.

[23] E. Liotti, C. Arteta, A. Zisserman, A. Lui, V. Lempitsky, and P. S. Grant, "Crystal nucleation in metallic alloys using X-ray radiography and machine learning," Sci. Adv., vol. 4, no. 4, p. eaar4004, Apr. 2018.

[24] Y. Jia, H. Huang, Y. Fu, G. Zhu, D. Shu, B. Sun, and D. H. StJohn, "An in situ investigation of the solute suppressed nucleation zone in an $\mathrm{Al}-15 \mathrm{wt} \% \mathrm{Cu}$ alloy inoculated by Al-Ti-B," Scr. Mater., vol. 167, no. July, pp. 6-10, 2019.

[25] A. Bogno, H. Nguyen-Thi, G. Reinhart, B. Billia, and J. Baruchel, "Growth and interaction of dendritic equiaxed grains: In situ characterization by synchrotron X-ray 
radiography," Acta Mater., vol. 61, no. 4, pp. 1303-1315, 2013.

[26] M. Becker, S. Klein, and F. Kargl, "In-situ solute measurements with a laboratory polychromatic microfocus X-ray source during equiaxed solidification of an Al-Ge alloy," Scr. Mater., vol. 124, pp. 34-37, Nov. 2016.

[27] D. A. Porter, K. E. Easterling, and M. Y. Sherif, Phase transformations in metals and alloys. CRC Press, 2009.

[28] S. Feng, E. Liotti, A. Lui, M. D. Wilson, T. Connolley, R. H. Mathiesen, and P. S. Grant, "In-situ X-ray radiography of primary Fe-rich intermetallic compound formation," Acta Mater., vol. 196, pp. 759-769, Sep. 2020.

[29] W. . Tiller, K. . Jackson, J. . Rutter, and B. Chalmers, "The redistribution of solute atoms during the solidification of metals," Acta Metall., vol. 1, no. 4, pp. 428-437, Jul. 1953.

[30] H. Wang, B. Cai, M. J. Pankhurst, T. Zhou, Y. Kashyap, R. Atwood, N. Le Gall, P. Lee, M. Drakopoulos, and K. Sawhney, "X-ray phase-contrast imaging with engineered porous materials over $50 \mathrm{keV}$," J. Synchrotron Radiat., vol. 25, no. 4, pp. 1182-1188, 2018.

[31] T. Nelson, B. Cai, N. Warnken, P. D. Lee, E. Boller, O. V. Magdysyuk, and N. R. Green, "Gravity effect on thermal-solutal convection during solidification revealed by fourdimensional synchrotron imaging with compositional mapping," Scr. Mater., vol. 180, pp. 29-33, Apr. 2020.

[32] Y. Kobayashi, K. Dobara, H. Todoroki, C. Nam, K. Morishita, and H. Yasuda, "In-situ measurements of solute partition coefficients between solid and liquid phases in $\mathrm{Fe}-\mathrm{Cr}-$ Ni-Mo-Cu alloys during solidification," ISIJ Int., vol. 60, no. 2, pp. 276-285, 2020.

[33] E. Liotti, A. Lui, T. Connolley, I. P. Dolbnya, K. J. S. Sawhney, A. Malandain, M. D. Wilson, M. C. Veale, P. Seller, and P. S. Grant, "Mapping of multi-elements during melting and solidification using synchrotron X-rays and pixel-based spectroscopy," Sci. Rep., vol. 5, p. 6, 2015.

[34] M. C. Veale, P. Seller, M. Wilson, and E. Liotti, "HEXITEC: A High-Energy X-ray Spectroscopic Imaging Detector for Synchrotron Applications," Synchrotron Radiat. News, vol. 31, no. 6, pp. 28-32, 2018.

[35] F. Abt, M. Boley, R. Weber, T. Graf, G. Popko, and S. Nau, "Novel X-ray System for insitu Diagnostics of Laser Based Processes - First Experimental Results," Phys. Procedia, vol. 12, pp. 761-770, Jan. 2011.

[36] T. Yamada, T. Shobu, A. Nishimura, Y. Yonemoto, S. Yamashita, and T. Muramatsu, "Insitu X-ray observation of molten pool depth during laser micro welding," J. Laser Micro Nanoeng., vol. 7, no. 3, pp. 244-248, 2012.

[37] C. L. A. Leung, S. Marussi, R. C. Atwood, M. Towrie, P. J. Withers, and P. D. Lee, "In situ X-ray imaging of defect and molten pool dynamics in laser additive manufacturing," Nat. Commun., vol. 9, no. 1, p. 1355, Dec. 2018.

[38] C. L. A. Leung, S. Marussi, M. Towrie, J. del Val Garcia, R. C. Atwood, A. J. Bodey, J. R. Jones, P. J. Withers, and P. D. Lee, "Laser-matter interactions in additive manufacturing of stainless steel SS316L and 13-93 bioactive glass revealed by in situ Xray imaging," Addit. Manuf., vol. 24, pp. 647-657, Dec. 2018.

[39] L. Aucott, H. Dong, W. Mirihanage, R. Atwood, A. Kidess, S. Gao, S. Wen, J. Marsden, S. Feng, M. Tong, T. Connolley, M. Drakopoulos, C. R. Kleijn, I. M. Richardson, D. J. Browne, R. H. Mathiesen, and H. V. Atkinson, "Revealing internal flow behaviour in arc welding and additive manufacturing of metals," Nat. Commun., vol. 9, no. 1, p. 5414, Dec. 2018. 
[40] Advanced Photon Source, "APS Upgrade Project Final Design Review Report - 2019," 2019.

[41] Diamond Light Source, "Diamond-II: Conceptual Design Report," 2019.

[42] H. J. Kirkwood, M. D. de Jonge, O. Muránsky, F. Hofmann, D. L. Howard, C. G. Ryan, G. van Riessen, M. R. Rowles, A. M. Paradowska, and B. Abbey, "Simultaneous X-ray diffraction, crystallography and fluorescence mapping using the Maia detector," Acta Mater., vol. 144, pp. 1-10, Feb. 2018.

[43] M. D. Wilson, L. Dummott, D. D. Duarte, F. H. Green, S. Pani, A. Schneider, J. W. Scuffham, P. Seller, and M. C. Veale, "A $10 \mathrm{~cm} \times 10 \mathrm{~cm}$ CdTe Spectroscopic Imaging Detector based on the HEXITEC ASIC," J. Instrum., vol. 10, no. 10, p. P10011, Oct. 2015. 